\title{
Supraventricular Tachycardia: An Unusual Side Effects of Cefuroxime
}

\author{
Yeap Boon Tat ${ }^{1 *}$, Yeoh Boon Seng ${ }^{2}$, Rajesh Kumar Muniandy ${ }^{1}$
}

\author{
1 Department of Medicine, \\ Faculty of Medicine and Health Sciences, \\ Universiti Malaysia Sabah, \\ Kota Kinabalu, Sabah, Malaysia \\ ${ }^{2}$ Department of Physiology, \\ School of Medical Sciences, \\ Health Campus, Universiti Sains Malaysia, \\ Kubang Kerian, Kelantan, Malaysia
}

\section{*Corresponding author's email: yeapboontat@gmail.com}

Received: 28 January 2018

Accepted: 24 December 2018

How to Cite:

Tat, Y., Boon Seng, Y., \& Muniad, R. (2019). Supraventricular tachycardia: An unusual side effects of cefuroxime. Borneo Journal of Medical Sciences (BJMS), 13 (2), 35-38. Retrieved from https://jurcon.ums.edu.my/ojums/index.php/ bjms/article/view/1134

Keywords: supraventricular

tachycardia, cefuroxime, anaphylaxis

\section{ABSTRACT}

Anaphylactic reaction towards antibiotics is common during anaesthesia. It may present as bronchospasm, hypotension, desaturation, or urticarial. However it is uncommon for anaphylaxis reaction to present only as supraventricular tachycardia (SVT). This is a rare interesting case report on a 23-year-old healthy man whose anaesthetic categorization is American Society of Anaesthesiologist (ASA) 1, developed supraventricular tachycardia (SVT) towards intravenous cefuroxime, perioperatively. His condition resolved with carotid sinus massage. No pharmacological interventions were used. His skin prick intradermal tests showed allergies towards cefuroxime, cefazoline and cefoperazone. The patient subsequently underwent.

\section{INTRODUCTION}

Cefuroxime is a broad spectrum second generation cephalosporin antibiotics that works widely on Gram positive and Gram negative organisms. It has bactericidalactivities by inhibiting the synthesis of bacterial cell wall. Common side effects of cefuroxime are nausea, vomiting, giddiness and abdominal pain which occur in a range of between 3 to $4 \%$ of the population treated ${ }^{1}$. The incidence of skin rashes was reported to be about $1 \%$ of the population ${ }^{2}$. It is commonly used in the operating theatre as prophylaxis against Gram positive bacteria in orthopaedics surgeries with implants. Prompt and early recognition 
of signs of severe anaphylaxis reaction of this drug is vital to reduce peri-operative morbidity and mortalities.

\section{CASE PRESENTATION}

A 23-year-old man, who had a closed fracture of right tibia and fibula due to a motor vehicle accident two days ago, was slated for an elective interlocking nail (ILN) insertion. He is an ASA 1 young man with no morbidities and no known drug or food allergies. His blood investigations prior to surgery were normal. We inserted an epidural catheter at the third and fourth inter space (L3 - L4) level with ease for intra and post-operative analgesia. Lidocaine $2 \% 3 \mathrm{ml}$ was used to anaesthetize the skin and the catheter was anchored at $9 \mathrm{~cm}$ in the epidural space. After that, the patient's trachea was intubated with a size 8 endotracheal tube. Drugs used for induction of anaesthesia were fentanyl, propofol and rocuronium as per ideal body weight. The patient was haemodynamically stable during induction and intubation.
A prophylactic dose of IV Cefuroxime 1.5 gram, diluted into a $20-\mathrm{ml}$ syringe, was prepared. $2 \mathrm{ml}$ of the drug was slowly given to the patient after intubation. After 2 minutes, there was a sudden formation of skin wheals on the left arm and chest, at the same site of the intravenous cannula. There was also abrupt rise of the heart rate from 70 beats per minute to a range of $190-200$ beats per minute. On the cardiac monitor, there were narrow complex supraventricular tachycardia (SVT) with regular rhythm and $P$ waves were identified. However, the patient's blood pressure was normal at range of $95-110$ / 60 $80 \mathrm{mmHg}$. The oxygen saturation maintained at $100 \%$ under $\mathrm{FiO} 2$ of $40 \%$ with no rhonchi heard upon auscultation of the lungs. The peak airway pressure maintained at $15-17 \mathrm{cmH}_{2} \mathrm{O}$. The patient was attended immediately and right carotid sinus massage was initiated. The heart rate responded by the gradual decrease to 65 - 70 beats per minute, after 10 minutes of initiation. Other haemodynamic parameters were stable. No pharmacological treatments were used to treat the tachycardia.

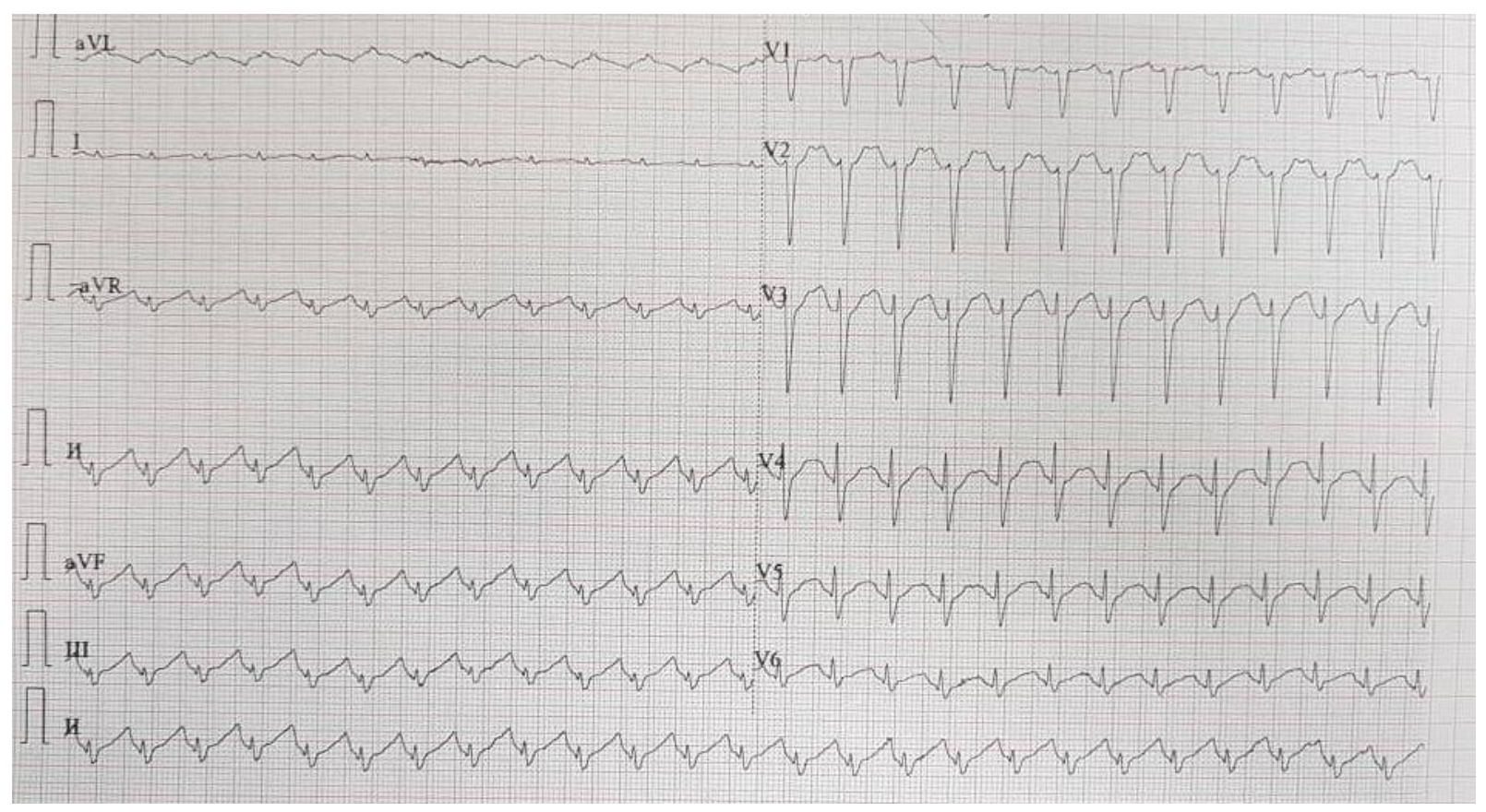

Figure 1 ECG of the patient intraoperatively showing SVT 
The surgical procedure was abandoned with a possibility of anaphylactic reactions to IV Cefuroxime. The patient was extubated well and remained haemodynamically stable in the recovery room. He was then transferred to the ward. The patient was referred to the hospital's pharmacist team and an intra dermal skin prick test was performed on the patient 4 weeks later with propofol, fentanyl, rocuronium and cefuroxime. It showed a positive reaction only towards cefuroxime, cefazolin and cefoperazone. There were no reactions towards other antibiotics, anaesthetic agents or opioids. The patient subsequently underwent.

\section{DISCUSSION}

Anaphylaxis is a severe, life-threatening, generalized systemic hypersensitivity reaction. Intraoperative anaphylaxis is a common phenomenon. However the exact incidences of anaesthesia-related anaphylaxis vary between 1 in 3,500 to 1 in 20,000 cases $^{3,4}$. They are commonly caused by muscle relaxants (60\%), latex hypersensitivity (20\%) and antibiotics $(15 \%)^{5}$. Of all the muscle relaxants, rocuronium has the highest incidence of anaphylaxis reactions ${ }^{6}$. However, from our local clinical anaesthetic experiences, rocuronium has the least likelihood to develop anaphylaxis reaction as compared to suxamethonium or atracurium.

S. Mali mentioned that antibiotics are third most common cause of intraoperative anaphylaxis ${ }^{7}$. Among the antibiotics, penicillin and cephalosporin group of drugs as the main culprit at $70 \%{ }^{5}$. This is due to both groups of drugs share the common beta-lactam ring. Compared to its common use in antibiotic prophylaxis, the sensitization rate seems to be low. The potential risk of anaphylactic reactions due to cephalosporin antibiotic is well documented.

The clinical features of anaesthesiarelated anaphylaxis are hypotension, tachycardia or bradycardia, occurring in 10\% of anaphylaxis reactions ${ }^{5}$. Besides that, other features include skin flushing and cutaneous rashes, bronchospasm, hypoxemia and cardiac arrest. These signs usually occur within a few minutes of exposure but may be delayed to after an hour.

Isolated case of second-generation cephalosporin (cefotiam) induced cardiomyopathy during anaesthesia was reported $^{8}$. However, the prevalence of supraventricular tachycardia (SVT) secondary to cephalosporin administration is largely unknown, but its role as potential cause of anaphylactic shock is probably underestimated.

SVT is a type of tachydysrhythmia that arises from above the level of Bundle of His. The common cause of SVT in a structurally normal heart is Atrioventricular Nodal Reentry Tachycardia (AVNRT) $)^{9}$. It is typically paroxysmal and may occur spontaneously or upon provocation. The heart rate usually ranges between 140 - 280 beats per minute and is regular in nature ${ }^{10}$. The QRS complex is usually $<0.12$ seconds and $P$ waves are visible. AVNRT is usually well-tolerated and rarely life-threatening in patients with concurrent heart disease. It may stop spontaneously or continue until medical therapy is sought. Haemodynamically-stable patients are treated by vagal manoeuvres, intravenous adenosine, diltiazem, or verapamil, whereas haemodynamically unstable patients are treated by cardioversion ${ }^{11}$.

In this case, the patient developed SVT and skin redness with wheals on the left arm, ipsilateral to the injection site, two minutes after the administration of IV Cefuroxime test dose. Haemodynamically, he was stable throughout. The SVT resolved spontaneously with application of right-sided carotid sinus massage. This is a typical presentation of anaesthesia-related anaphylaxis to antibiotics. As the patient's haemodynamic was relatively stable during and after induction of anaesthesia, the cause of the SVT was due to 
IV Cefuroxime, as confirmed by the skin prick test post-operatively. A raised serum tryptase level will be handy in diagnosing anaphylaxis reactions ${ }^{12,13}$. Our centre was not able to perform blood test for serum tryptase levels due to logistics issues.

\section{CONCLUSION}

This was the case of supraventricular tachycardia which was induced by intravenous cefuroxime. All anaesthetists should note with care that even with a considerably safe drug such as Cefuroxime, a severe cardiorespiratory collapse can still occur. Prompt recognition and treatment of an anaphylactic reaction may prevent its progression to a severe irreversible bronchospasm, laryngeal oedema, cardiac dysfunction, irreversible arrhythmias and cardiac arrest.

\section{CONFLICT OF INTEREST}

The authors declare that they have no competing interests in publishing this case.

\section{CONSENTS}

Written informed consent was obtained from the patient to publish the case with its related pictures. A copy of the written consent is available for review by the Chief Editor.

\section{REFERENCES}

1. Gold B., Rodriguez WJ. (1983). Cefuroxime: Mechanisms of action, antimicrobial activity, pharmacokinetics, clinical applications, adverse reactions and therapeutic indications. Pharmacotherapy: The Journal of Human Pharmacology and Drug Therapy 3 (2P1): $82-100$.

2. Norrby SR. (1987). Side effects of cephalosporins. Drugs 34 (2): 105 - 120.
3. Fisher MM, Baldo BA. (1993). The incidence and clinical features of anaphylactic reactions during anesthesia in Australia. Ann Fr Anesth Reanim 12 (2): 97 - 104.

4. Laxenaire MC. (1999). Epidemiology of anesthetic anaphylactoid reactions. Fourth multicenter survey (July 1994 - December 1996). Ann Fr Anesth Reanim 18 (7): 796 809.

5. Harper NJ, Dixon T, Dugue P, Edgar DM, Fay A, Gooi HC. (2009). Suspected anaphylactic reactions associated with anaesthesia. Anaesthesia 64 (2): 199 - 211. doi: 10.1111/j.1365-2044.2008.05733.x

6. Cho YJ, Ju JW, Sim H, Lee JH, Hong DM, Kim TK, Jeon Y. (2016). Intraoperative anaphylaxis to neuromuscular blocking agents: The incidence over 9 years at two tertiary hospitals in South Korea: A retrospective observational study. Eur J Anaesthesiol 33 (5): 368 - 378. doi: 10.1097/ EJA.0000000000000373

7. Mali S. (2012). Anaphylaxis during the perioperative period. Anesth Essays Res 6 (2): 124 - 133. doi: 10.4103/0259-1162.108286

8. Suk EH, Kim DH, Kweon TD, Na SW, Shin JA. (2009). Stress-induced cardiomyopathy following cephalosporin-induced anaphylactic shock during general anesthesia. Canadian Journal of Anesthesia/Journal canadien d'anesthésie 56 (6): 432 - 436. doi: 10.1007/ s12630-009-9083-0

9. Kumar DS, Dewland T, Balaji S, Henrikson CA. (2017). How to approach difficult cases of AVNRT. Curr Treat Options Cardiovasc Med 19 (5): 34. doi: 10.1007/s11936-017-0531-9

10. Fox DJ, Tischenko A, Krahn AD, Skanes AC, Gula LJ, Yee RK, Klein GJ. (2008). Supraventricular tachycardia: Diagnosis and management. Mayo Clin Proc 83 (12): 1400 1411. doi: 10.1016/S0025-6196(11)60791-X

11. Al-Zaiti SS, Magdic KS. (2016). Paroxysmal supraventricular tachycardia: Pathophysiology, diagnosis, and management. Crit Care Nurs Clin North Am 28 (3): 309 - 316. doi: 10.1016/j. cnc.2016.04.005

12. AseroR,FarioliL,PastorelloEA.(2012). Baseline serum tryptase levels and adverse reactions to injection specific immunotherapy with airborne allergens: Is there a relationship? Int Arch Allergy Immunol 158 (3): 276 - 280. doi: $10.1159 / 000331315$

13. Ordoqui E, Zubeldia JM, Aranzabal A, Rubio M, Herrero T, Tornero P, Baeza ML. (1997). Serum tryptase levels in adverse drug reactions. Allergy 52 (11): 1102 - 1105. 